\title{
ANALYSES OF METALLIC FIRST MIRROR SAMPLES AFTER LONG TERM PLASMA EXPOSURE IN TORE SUPRA
}

\author{
M. LIPA ${ }^{* 1}$, B. SCHUNKE ${ }^{1}$, CH. GIL ${ }^{1}$, J. BUCALOSSI $^{1}$, V. S. VOITSENYA ${ }^{2}$, V. \\ KONOVALOV ${ }^{2}$, K. VUKOLOV ${ }^{3}$, M. BALDEN ${ }^{4}$, G. DE TEMMERMAN ${ }^{5}$, P. OELHAFEN ${ }^{5}$, \\ A. LITNOVSKY ${ }^{6}$, P. WIENHOLD ${ }^{6}$ \\ 1 Association Euratom-CEA, CEA/DSM/DRFC, CEA-Cadarache, 13108 Saint-Paul-Lez- \\ Durance, France \\ 2 Institute of Plasma Physics, NSC KIPT, Akademichna St. 1, 61108 Kharkov, Ukraine \\ 3 NFI RRC Kurchatov Institute, Moscow, Russia \\ 4 Max-Planck-Institut für Plasmaphysik, Boltzmannstr. 2, D-85748 Garching, Germany \\ 5 Institut für Physik, Universität Basel, Klingelbergstrasse 82, CH-4056 Basel, Switzerland \\ 6 Institut für Plasmaphysik, Forschungszentrum Jülich, D-42425 Jülich, Germany
}

Abstract: Metallic mirrors are foreseen in ITER diagnostic systems as optical elements directly viewing the plasma radiation. In the frame of an EFDA contract, metallic mirror samples have been exposed for long pulse plasma discharges in Tore Supra (TS) in order to investigate surface modifications caused by erosion and re-deposition processes. Three different materials have been selected: mono-crystalline molybdenum (mc-Mo), polycrystalline stainless steel $(\mathrm{SS})$ and copper $(\mathrm{Cu})$. The mc-Mo samples showed after TS exposure almost no surface roughness modifications and the lowest net-erosion. A slight reflectivity reduction, most pronounced in the near $\mathrm{UV}$, is attributed to light absorption in a thin carbon deposit. $\mathrm{Cu}$ mirrors showed by far the highest surface roughness, erosion and diffusive reflectivity. Comparative laboratory glow discharge experiments with virgin reference samples and numerical simulations of erosion/deposition confirm the dominant contribution of conditioning procedures to erosion of mirrors exposed (without shutter protection) in Tore Supra.

Keywords: First mirrors, mirror erosion and re-deposition, Tore Supra mirror exposure

\footnotetext{
* Corresponding author : Tel. :+33-4-4225-4658; fax: +33-4-4225-4990; E-mail address: manfred.lipa@cea.fr
} 


\section{Introduction}

First mirrors will be the plasma viewing components of optical diagnostic systems in ITER. Attention focus on two processes, which can lead to a degradation of the mirror optical properties, namely:

-sputtering by charge exchange (CX) neutrals and ions during plasma operation and during conditioning procedures such as discharge cleaning, which leads to erosion; -deposition of material eroded from the first wall, which leads to surface contamination [1]. In the frame of an EFDA contract, metallic mirror samples (22 mm in diameter, $4 \mathrm{~mm}$ thick) of three different materials: mono-crystalline molybdenum (mc-Mo), polycrystalline stainless steel (SS) and copper $(\mathrm{Cu})$ - were installed in TS for long-term exposure during the experimental campaign 2003-2004 [2]. All mirrors, including samples for reference measurements, were supplied by IPP-NSC KIPT Kharkov, where the surface of the monocrystal molybdenum samples was oriented to (110) with a divergence of $\sim 30$ minutes. The stainless steel samples were made from a polycrystalline material developed in the USSR as an analogue of the AISI 316 steel (C0.04Cr16Ni11Mo3Ti). The copper samples were made from a polycrystalline oxygen free copper.

\section{Experimental layout and operation conditions}

Two mirrors of each material have been installed on the high field side of the TS plasma vessel near the observation sector Q3A. They were located at a poloidal angle of $\Theta=7.6^{\circ}$ (sample set B-D, B-B, B-G) and $15.4^{\circ}$ (sample set A-D, A-B, A-G) out of the equatorial plane $(\mathrm{R}=2421, \mathrm{r} \sim 880)$ and positioned approximately $140 \mathrm{~mm}$ from the LCFS (last closed flux surface). The reflecting surfaces of all mirrors were oriented parallel (not inclined) to the toroidal direction and their surfaces located radially $5 \mathrm{~mm}$ behind the assembly structure 
(without the possibility of a shutter protection), which allowed contact cooling via the first wall panels (Fig.1).

During about one year of exposure, about 1400 plasma pulses (mainly $\mathrm{D}_{2}$ ) with plasma current Ip $\geq 200 \mathrm{kA}\left(\mathrm{n}_{\mathrm{e} 0} \sim 2-410^{19} \mathrm{~m}^{-3}\right)$ have been performed with a cumulative pulse length of 26000 s $(7 \mathrm{~h} 10)$. The integrated injected energy in TS was roughly 37 Gigajoules (GJ) composed of $\sim 13$ GJ ohmic, $\sim 22$ GJ lower hybrid and $\sim 2$ GJ ICRH. In addition wall conditioning by glow discharges in $\mathrm{He}\left(\mathrm{t}=362 \mathrm{~h}, \mathrm{I}=7 \mu \mathrm{A} / \mathrm{cm}^{2} ; \mathrm{U}_{\text {anode }}=300 \mathrm{~V}, \mathrm{p}=0.3 \mathrm{~Pa}\right)$, in $\mathrm{D}_{2}$ $\left(\mathrm{t}=606 \mathrm{~h}, \mathrm{I}=7 \mu \mathrm{A} / \mathrm{cm}^{2}, \mathrm{U}_{\text {anode }}=400 \mathrm{~V}, \mathrm{p}=0.3 \mathrm{~Pa}\right)$ and $13 \mathrm{~h}$ of boronization, in between plasma operation, have been performed during this exposure time. A major water leak of an actively cooled in-vessel component in September 2003 led to water drop projections on the mirror surface (the mirrors have not been cleaned after that, before further exposure).

Baking cycles of the vacuum vessel structure were performed at temperatures around $200{ }^{\circ} \mathrm{C}$. 2D-thermohydraulic finite element calculations of the mirror sample assemblies showed temperatures not exceeding $\sim 150{ }^{\circ} \mathrm{C}$ for a typical "Gigajoule" operation scenario $(\sim 1 \mathrm{MW}$ radiated plasma power loss during $360 \mathrm{~s}$ of pulse length).

\section{Post exposure mirror analyses}

Surface roughness and 3D surface profiles have been measured by confocal microscopy (CM). Surface topography and chemical analyses were performed by SEM imaging, EDX, XPS and SIMS. Reflectivity measurements were carried out using a spectrophotometer equipped with an integrating sphere operating in the spectral range of 250-2500 nm. Optical constants $\boldsymbol{n}$ (refraction index) and $\boldsymbol{k}$ (extinction coefficient) were measured by ellipsometry in the range between $300-850 \mathrm{~nm}$. All measurements were compared with results obtained on virgin reference samples. 


\section{1 mc-molybdenum (A-D, B-D)}

The surface roughness $\mathrm{Ra} \sim 0.7 \mathrm{~nm}(\mathrm{Ra}=$ arithmetical mean value of the amounts of the ordinate values $\mathrm{Z}(\mathrm{x})$ within an individual measuring distance Ir- ISO 4287: 1997) of the mcmolybdenum samples showed almost no deterioration and, within the accuracy of the $\mathrm{CM}$ measurement method, a net-erosion depth of roughly $0.12 \mu \mathrm{m}$.

SEM imaging revealed an almost unchanged surface. Some geometrical not regular shaped microparticle structures on the surface can be seen; their density is similar than on SS but lower than on $\mathrm{Cu}$ surfaces. The composition of these particles measured by EDX is dominated by $\mathrm{C}$ and $\mathrm{O}$ (Fig. 2). The elongated form of the particles may indicate shaping by water drops due to an in-situ water leak (water projection on mirror surface), which occurred during sample exposition. XPS measurements on the mirror surface show C1s, Mo $3 \mathrm{~d}, \mathrm{Fe} 2 \mathrm{p}$ and $\mathrm{O}$ 1s lines. The molybdenum line shows a triplet revealing the presence of the bulk material coated with a thin molybdenum trioxide layer (the mirror samples were air exposed after removal from TS). SIMS surface analyses show carbon deposits enriched with hydrogen, deuterium, boron and oxygen (Fig. 3). In order to estimate the deposited film thickness on the exposed mc-Mo mirror sample, the SIMS facility was calibrated using a Dektak-6M mechanical profiler. A carbon deposit thickness of $12 \mathrm{~nm}$ was estimated. This thickness is also in some way confirmed by colorimetry: 10-15 nm thick deposits are still transparent, but 20-25 nm thick show already interference colors seen by the unaided eye [3].

The total and the specular reflectivity show a slight decrease compared to the virgin sample. Specular values are compared to SS and $\mathrm{Cu}$ in Fig. 4. This decrease is more pronounced in the UV region. The diffuse reflectivity remains very low $(<2 \%)$. Since the diffusive component of the reflectivity is linked to the roughness of the material, we can deduce that the roughness has not evolved sensibly during exposure (plasma and conditioning procedures), which has 
been confirmed by surface roughness measurements. We can assume that the decrease of the specular reflectivity is due to light absorption in the thin layer of carbon deposited.

\subsection{Stainless steel (A-B, B-B)}

The exposed stainless steel sample showed a surface roughness Ra of $5.7 \mathrm{~nm}$ (however, virgin reference samples varied between 1.6 to $8 \mathrm{~nm}$ ) and a net-erosion depth of roughly $0.22 \mu \mathrm{m}$. A low scale grain-to-grain relief and in-grain topography is visible in SEM. The density of microparticles for the SS surface is similar to that for the Mo sample and, therefore, lower as for $\mathrm{Cu}$. Again the composition of these particles is dominated by $\mathrm{C}$ and $\mathrm{O}$. Traces of other impurities such as Mo and Si are observed. SIMS analyses of the mirrors show surface contaminations with oxygen and boron impurities. Also hydrogen and deuterium is observed, while it was difficult to make solid conclusions for the existence of a deposited layer.

A decrease of both, the total and specular reflectivity (Fig. 4), is observed after exposure. Diffuse reflectivity is higher especially in the UV region $(<6 \%)$. Therefore the drop of specular reflectivity may be attributed to some extend to erosion processes.

\subsection{Copper (A-G, B-G)}

The most dramatic surface change, which is clearly visible by the unaided eye, were observed on the copper mirrors with $\mathrm{Ra}$ of $47-69 \mathrm{~nm}$ (virgin reference sample varied between 7 to 8 nm) and an important erosion depth of roughly $2.33-2.6 \mu \mathrm{m}$ (Fig.5).

A strong grain-to-grain relief and in-grain topography is visible in SEM. The grain-to-grain steps seems to be partly covered by a deposited layer, which shows an additional erosion topography. The original topography has drastically changed (Fig. 6). The microparticle density is much higher than for Mo and SS specimens, very different in form and size and depends on grain orientation. The composition of some of these particles with size up to $1 \mu \mathrm{m}$ 
is dominated by $\mathrm{C}$ and $\mathrm{O}$. XPS spectra show impurities like carbon, boron, silicon and oxygen. Moreover a fitting procedure applied to the copper line reveals the presence of a layer of copper oxide. Copper is present in two states, as an oxide layer and as a bulk non-oxidized material (the mirror samples were also air exposed after removal from TS). SIMS analyses at the mirror center show contaminations with oxygen and boron impurities. Also hydrogen and a small amount of deuterium can be distinguished, while it was difficult to identify a deposited layer.

The diffuse reflectivity reaches extreme values of about $50 \%$ and, consequently, the specular component $\sim 40 \%$ at $800 \mathrm{~nm}$. Therefore, we can assume that the drop of specular reflectivity is mainly due to erosion processes. As for the other mirror materials, relative reflectivity measurements in the FIR $(119 \mu \mathrm{m})$ showed no significant modifications after exposure.

\section{Erosion estimations}

In order to verify the influence of erosion due to physical sputtering by ions, virgin reference mirror samples of the same fabrication batch have been exposed ex-vessel (EV) to $\mathrm{He}$ and $\mathrm{D}_{2}$ ions in a special laboratory chamber using the same glow electrode/mirror assembly configuration and similar discharge parameters than in TS (\$2). After each (He and additional $\mathrm{D}_{2}$ ) exposure period, surface roughness and profile measurements were performed by CM. From the synthesized 3D images of TS and EV exposed mirrors it can be seen that the eroded surfaces are not homogeneous and have no rotational symmetry. Note, that the mentioned erosion depths are obtained between the edge of the erosion area and its shadowed area and, therefore, may not represent correctly an average value. A variety of effects may have led to erosion pattern inhomogeneities (in TS as well as EV), such as: original surface imprecisions, field potential inhomogeneities, particle incidence ("configuration factor"), surface roughness, 
etc. The laboratory EV results normalized for TS conditioning procedures (exposure time, current density) are shown in Tab.1. In numerical simulations with the EIRENE code [4], CXS fluxes $\left(\sim 1.510^{20} \mathrm{~m}^{-2} \mathrm{~s}^{-1}\right)$ and particle energies near the mirror samples were calculated in order to estimate erosion by physical sputtering at normal incidence during plasma operation. These values were compared with estimated glow discharge erosion [5]. Detailed modeling is discussed in [2] and [6]. The tendency of calculation results confirm the experimental measurements in the sense, that erosion due to physical sputtering by ions during glow discharge wall conditioning (mirrors without shutter protection) dominates over erosion from CX neutrals during plasma operation (Table 1).

\section{Conclusion}

In the given exposure location the $\mathrm{CX}$ neutral fluxes and carbon re-deposition fluxes were minimized due to geometrical constraints of the TS configuration, thus also minimizing the effect of plasma operation on erosion and deposition. Nevertheless the long-term plasma exposure experiment of mirror samples in Tore Supra clearly demonstrate, that the mirror optical properties were degraded due to two opposite processes: (i) deposition of contaminating films (on Mo samples), which are, however, difficult to detect on SS and $\mathrm{Cu}$, (ii) sputtering by CX neutrals during plasma operation and especially ions during alternating long lasting glow discharges. Due to the long mirror exposure time in TS, without intermediate characterizations, it was extremely difficult to quantify correctly the interplay phenomena between mirror erosion and deposition mechanisms. However from comparative EV glow discharge experiments and erosion calculations one can conclude, that mirror erosion in TS was dominated by wall conditioning glow discharges. 


\section{References}

[1] V. Voitsenya, A. Costley, V. Bandourko, et al., Rev. Sci. Instr. 72 (2001) 475-482.

[2] M. Lipa, B. Schunke, Ch. Gil et al., First mirror study in Tore Supra, EFDA ref. TW2TPDS-DIADEV-D02, Final report, January 2005.

[3] P. Wienhold et al., Nucl. Instr. and Meth. in Phys. Res. B 94, (1994), 503-510.

[4] D. Reiter, The EIRENE Code, User Manual, Technical Report Juel-2599, FZJ (1992).

[5] W. Eckstein, C. Garcia-Rosales, J. Roth et al., Sputtering Data, Technical Report IPP 9/82, MPI Garching (1993).

[6] B. Schunke, M. Lipa et al., Modelling of the plasma conditions for the mirror exposure study in Tore Supra, accepted for 32nd EPS (2005), Tarragona, Spain. 
Fig. 1 Location of the first mirror samples on Tore Supra vessel wall at high field side.

Fig. 2 EDX spectra of a microparticle structure (thick line) and averaged over a larger area (thin line/grey) of mc-Mo A-D mirror sample.

Fig. 3 SIMS spectrum of mc-molybdenum (A-D) mirror sample exposed in TS.

Fig. 4 Specular reflectivity versus wavelength of exposed (mc-Mo, SS, Cu) and virgin reference samples.

Fig. 5 Confocal microscopy synthesised 3 D image of exposed copper (A-G) mirror sample of $22 \mathrm{~mm}$ in diameter.

Fig. 6 SEM image of exposed copper (A-G) mirror sample. 


\begin{tabular}{|l|l|c|c|c|}
\hline $\begin{array}{l}\text { Exposed mirror } \\
\text { sample material }\end{array}$ & $\begin{array}{l}\text { EV norm. He }(362 \mathrm{~h}) \\
+\mathrm{D}_{2}(606 \mathrm{~h}) \\
\text { glow -erosion } \\
(\mu \mathrm{m})\end{array}$ & $\begin{array}{l}\text { Calcul. CXS - } \\
\text { erosion in TS } \\
(\mu \mathrm{m})\end{array}$ & $\begin{array}{l}\text { Calcul. total } \\
(- \text { CXS+glow }) \\
\text { erosion in TS } \\
(\mu \mathrm{m})\end{array}$ & $\begin{array}{l}\text { Net-erosion } \\
\text { measured in TS } \\
(\mu \mathrm{m})\end{array}$ \\
\hline Mc-Molybdenum & $\sim 0.061+0.25=0.31$ & $\sim 0.0317$ & $\sim 0.107$ & $\sim 0.12$ \\
\hline Stainless Steel & $\sim 0.3+0.17=0.47$ & $\sim 0.0379$ & $\sim 0.5185$ & $\sim 0.22$ \\
\hline Copper OFHC & $\sim 0.31+2.53=2.84$ & $\sim 0.0685$ & $\sim 1.0085$ & $\sim 2.68$ \\
\hline
\end{tabular}

Table 1 M. Lipa $1 / 6 \mathrm{pp}$

Table 1 Comparison between laboratory (ex-vessel-EV) erosion results normalized for TS conditioning procedures, calculated and TS net-erosion values. 


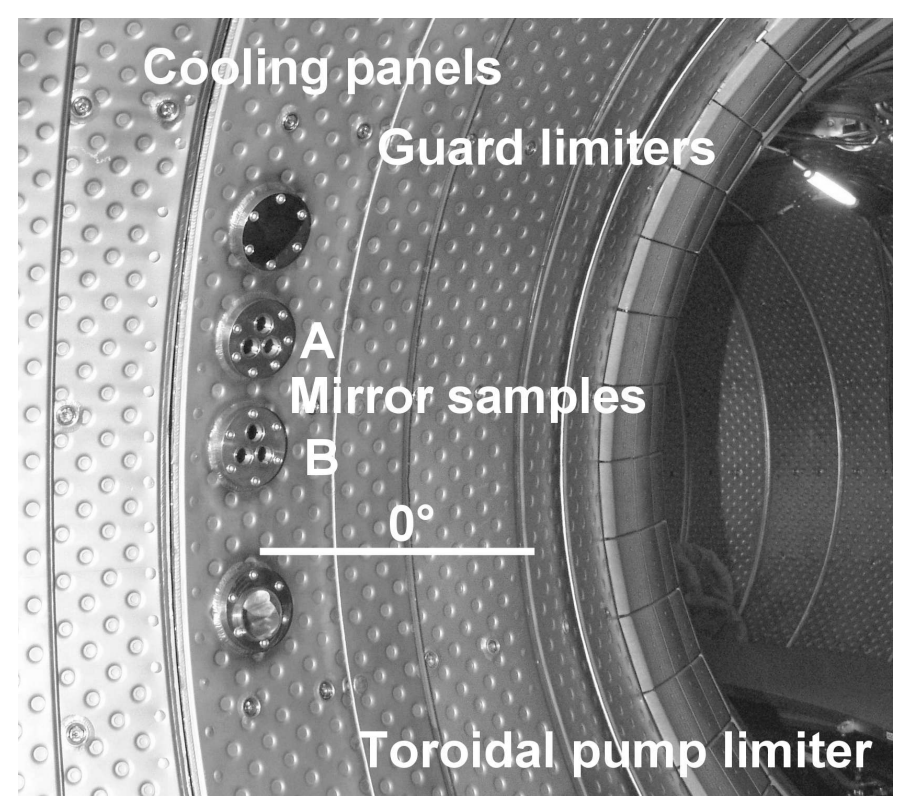

Fig. 1 M. Lipa $1 / 6 p p$ 


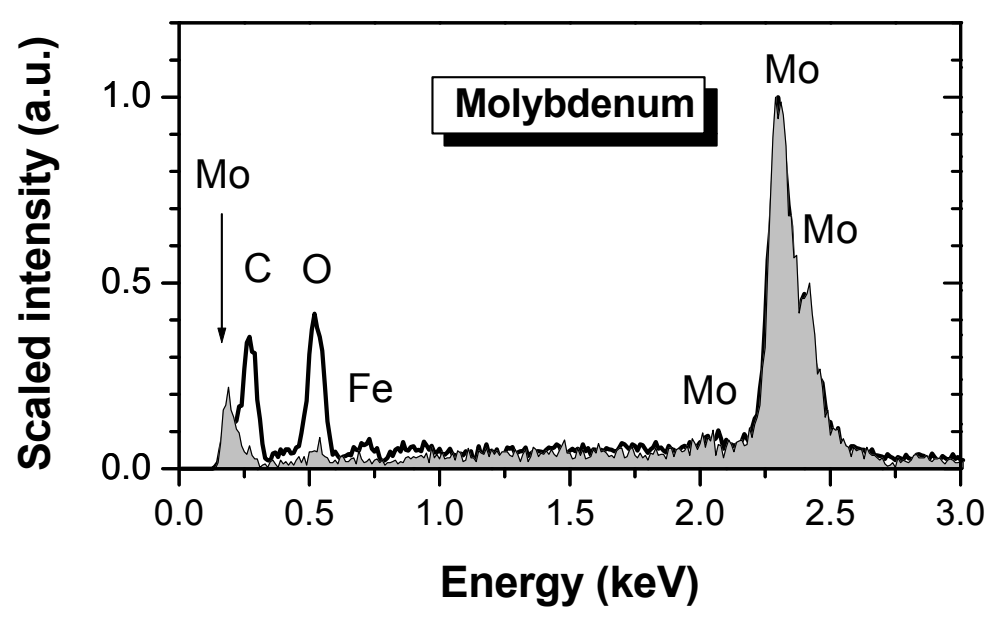

Fig. 2 M. Lipa 1/6 pp 


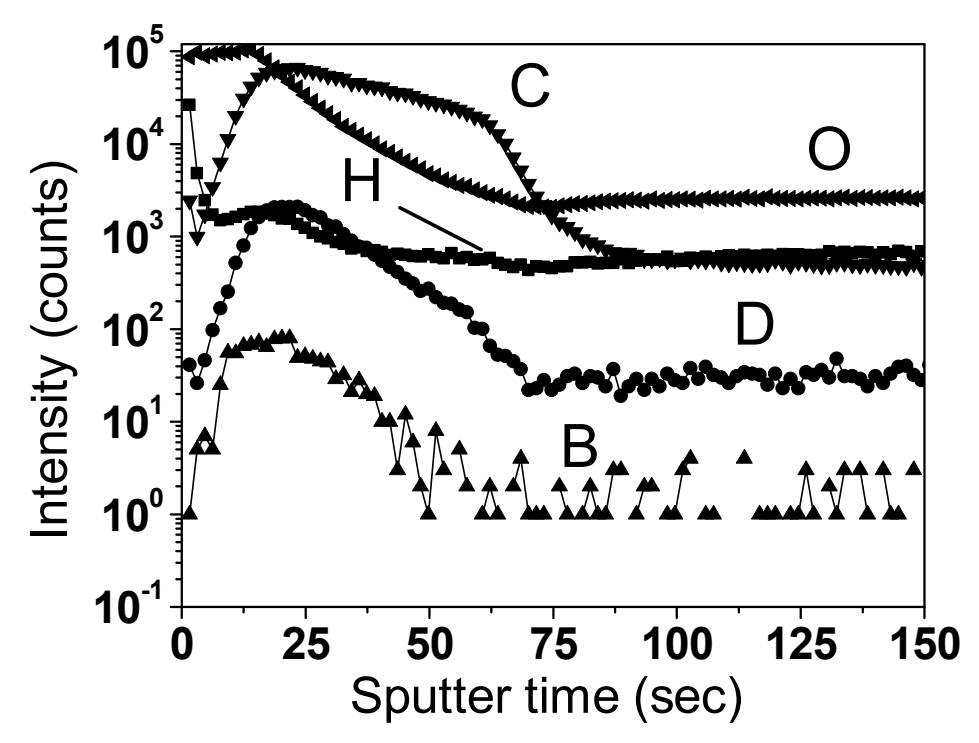

Fig. 3 M. Lipa 1/6 pp 


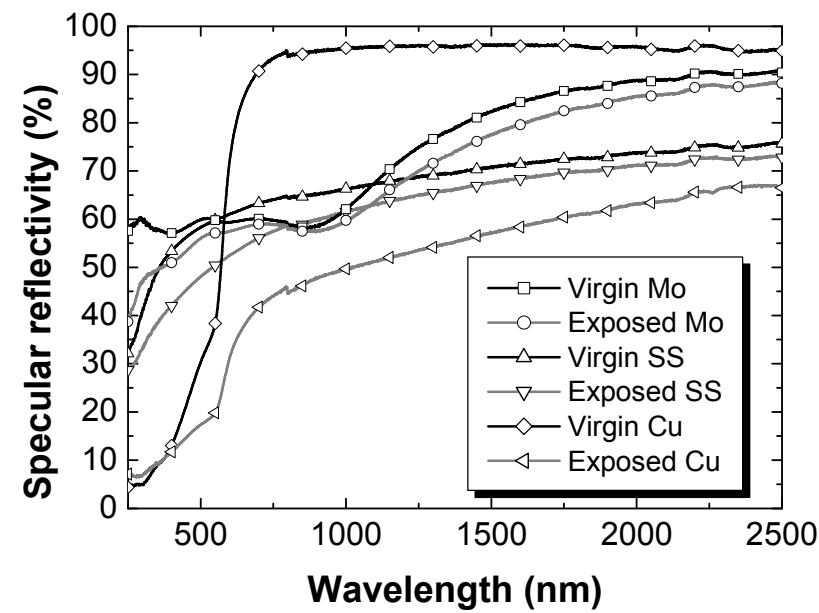

Fig. 4 M. Lipa $1 / 6 p p$ 
Alpha $=302^{\circ} \quad$ Beta $=45^{\circ}$

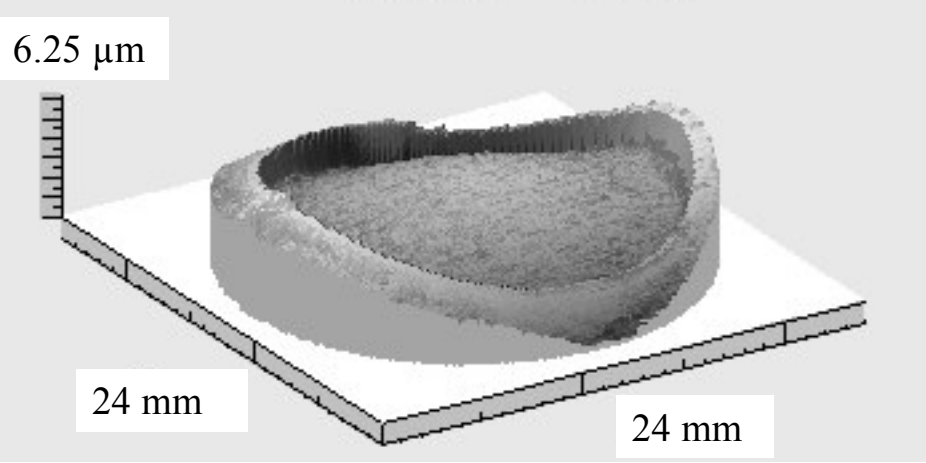

Fig. 5 M. Lipa $1 / 6 p p$ 


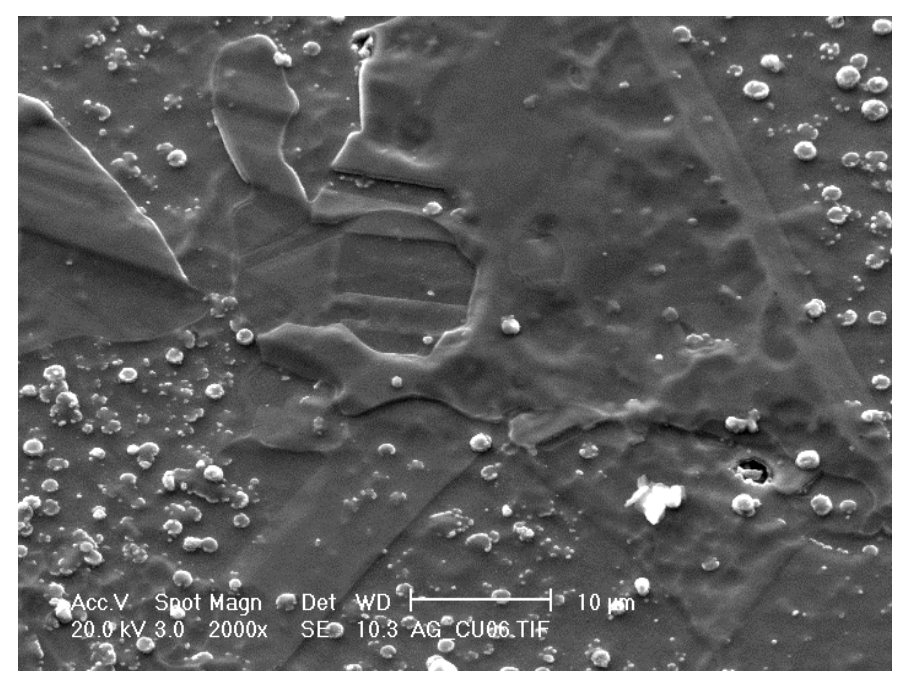

Fig. 6 M. Lipa 1/6 pp 